\title{
Failure of Fondaparinux in Autoimmune Heparin-Induced Thrombocytopenia
}

\author{
Michelangelo Sartori $^{1}$ Benilde Cosmi ${ }^{1}$ \\ ${ }^{1}$ Cardiovascular Department, Angiology and Blood Coagulation Unit, \\ S. Orsola-Malpighi University Hospital, Bologna, Italy \\ TH Open 2020;4:e305-e308.
}

\begin{abstract}
Address for correspondence Michelangelo Sartori, MD, PhD, U.O. di Angiologia e Malattie della Coagulazione, Azienda Ospedaliera di Bologna, Policlinico Sant'Orsola Malpighi, Pad. 2, Via Albertoni, 15, 40138 Bologna, Italy (e-mail: michelangelo.sartori@aosp.bo.it).
\end{abstract}

\begin{abstract}
Keywords

- heparin-induced thrombocytopenia

- rivaroxaban

- deep vein thrombosis

- fondaparinux

Heparin-induced thrombocytopenia (HIT) is an immune adverse reaction to heparin that is associated with life-threatening thrombotic complications. More rarely, HIT may begin after stopping of heparin or after flushes of heparin (autoimmune HIT). Fondaparinux has been proposed as a candidate treatment for HIT, but there are few data on its use in autoimmune HIT. An 86-year-old man with a history of diabetes mellitus, arterial hypertension, and hypercholesterolemia was admitted to our hospital for carotid endarterectomy. During surgery, only one heparin dose of 5,000 U was used. Platelet count started to decrease on the 11 th day after surgery. Since the patient was not receiving heparin treatment/prophylaxis, HIT was not suspected. On day 19 , platelet count was $61 \times 10^{3} / \mu \mathrm{L}$, and the patient was investigated for a diagnosis of HIT. Immunoglobulin (Ig)-G-specific enzyme-linked immunosorbent assay (ELISA) was positive and HIT was confirmed by a platelet aggregation test; fondaparinux $5 \mathrm{mg}$ once a day was started. During fondaparinux treatment, platelet count did not increase and a lower leg deep vein thrombosis occurred. Fondaparinux was stopped and rivaroxaban $15 \mathrm{mg}$ twice a day was started. Platelet count returned to base line after 10 days from fondaparinux withdrawal. There was no thrombotic event or bleeding complication during rivaroxaban treatment. Anecdotal evidence suggests risk of failure of fondaparinux treatment for autoimmune HIT and supports the use of rivaroxaban for treatment of HIT, justifying larger studies.
\end{abstract}

\section{Introduction}

Heparin-induced thrombocytopenia (HIT) is a prothrombotic condition that is associated with increased in vivo thrombin generation that needs to be treated with nonheparin anticoagulants. In the majority of cases (typical-onset HIT), after a mean of 4 to 6 days of heparin exposure, immunoglobulin (Ig)$\mathrm{G}$ antibodies against platelet factor 4 (PF4) bound to heparin develop. This is followed by the onset of the platelet count fall, and finally by thrombosis. ${ }^{2}$ More rarely, HIT may follow the exposure to heparin "flushes" or after stopping heparin. Such condition has been described and classified as "autoimmune HIT." 3 Often, autoimmune HIT patients have severe thrombo- cytopenia that may last for several weeks, and this is not a contraindication to anticoagulant therapy. ${ }^{3}$ Patients with autoimmune HIT require nonheparin anticoagulants, high therapeutic levels of anticoagulation are needed to control such hypercoagulable state. ${ }^{3}$ Recent guidelines recommend the use of danaparoid, argatroban, fondaparinux, or a direct oral anticoagulant for HIT treatment. ${ }^{4}$ Fondaparinux is commonly used in every day clinical practice for clinically suspected and confirmed acute HIT. In a German registry, no thrombotic complication occurred in HIT patients treated with fondaparinux, ${ }^{5}$ whereas some case reports have shown failure of fondaparinux anticoagulation for HIT with evidence of in vivo cross-reactivity HIT antibodies. ${ }^{6,7}$ Recently, rivaroxaban, received

March 19, 2020 accepted after revision May 11, 2020
DOI https://doi.org/

10.1055/s-0040-1713175. ISSN 2512-9465.
๑) 2020 Georg Thieme Verlag KG
Stuttgart · New York

License terms

(c) (i) 
an oral direct factor Xa inhibitor, has been proposed as a candidate for treatment of HIT because it seems to have a better safety profile than the aforementioned agents and it is administered orally by fixed dosing. ${ }^{8}$ As suggested by several case series, the administration of rivaroxaban may be adequate for thrombosis treatment and normalization of platelet count in patients with HIT $^{9}$ and with autoimmune HIT. ${ }^{3,6}$ Here, we report a case of autoimmune HIT, in which a new thrombosis occurred during fondaparinux treatment, whereas rivaroxaban was not associated with thrombotic events or bleeding complications.

\section{Case Description}

An 86-year-old man (weight: $74 \mathrm{~kg}$ ) with a history of diabetes mellitus, arterial hypertension, hypercholesterolemia was admitted to our hospital for carotid endarterectomy. He was on therapy with ramipril $5 \mathrm{mg}$ once a day, aspirin $100 \mathrm{mg}$ once a day, amlodipine $10 \mathrm{mg}$ once a day, omeprazole $20 \mathrm{mg}$ once a day, and simvastatin $40 \mathrm{mg}$ once a day. His medical history included a previous ischemic stroke and the presence of an infrarenal abdominal aortic aneurysm (diameter: $4.2 \mathrm{~mm}$ ) and a $70 \%$ stenosis of left internal carotid. During carotid endarterectomy a bolus of unfractionated heparin (UFH), dose of 5,000 $\mathrm{U}$, was used. Aspirin was continued during and after surgery. No prophylactic low molecular weight heparin was administered after surgery. As shown in the figure, in the morning before surgery his platelet count was $143 \times 10^{3} / \mu \mathrm{L}$ (day 0 : $\mathrm{D} 0$ ), platelet count decreased to $115 \times 10^{3} / \mu \mathrm{L}$ on D3. On D5, platelet count increased, and reached $138 \times 10^{3} / \mu \mathrm{L}$ on D7. Platelet count was not repeated till D11 when a decrease was observed $\left(110 \times 10^{3} / \mu \mathrm{L}\right)$. Since the patient was not receiving heparin treatment/prophylaxis, HIT was not initially suspected. On D16, platelet count was $91 \times 10^{3} / \mu \mathrm{L}$, the pretest clinical score ( 4 T's) for the diagnosis of HIT $^{10}$ was 4 , but HIT was still not suspected. On D18, the platelet count declined further to
$61 \times 10^{3} / \mu \mathrm{L}$ and the patient was therefore investigated for a diagnosis of delayed onset and persisting (autoimmune) HIT. A whole leg ultrasound did not show any deep vein thrombosis (DVT), 4T's score was 5 and, on D19, HIT was diagnosed on the basis of the presence of HIT antibodies. An IgG-specific ELISA (PF4-enhanced IgG, Immucor GTI Diagnostics, Inc.) was strongly positive ( $\mathrm{OD}=2.359)$. The presence of HIT was confirmed by a platelet aggregation test. Platelet aggregation was performed using a four channel Chrono-Log platelet aggregometer (model 540, Chrono-Log Corp., Havertown, Pennsylvania, United States): $10 \mu \mathrm{L}$ of UFH ( 1 and $100 \mathrm{IU} /$ $\mathrm{mL}$ final concentration) was added to the cuvette and the aggregation response was monitored for 20 minutes. ${ }^{11}$ The presence of HIT was confirmed if the aggregation was $>20 \%$ with $1 \mathrm{IU} / \mathrm{mL}$ UFH and completely inhibited or $<20 \%$ with $100 \mathrm{IU} / \mathrm{mL}$ UFH. The patient's serum induced rapid activation of platelets from two donors in the presence of $1 \mathrm{IU} / \mathrm{mL} \mathrm{UFH,}$ as well as in the absence of UFH, that was inhibited with $100 \mathrm{IU} / \mathrm{mL}$ UFH. He had renal insufficiency: serum creatinine was $1.2 \mathrm{mg} / \mathrm{dL}$ (estimated creatinine clearance with Cockcroft-Gault formula was $46 \mathrm{~mL} / \mathrm{min}$ ), and fondaparinux treatment was started. Given the absence of thrombosis and the presence of chronic kidney disease stage III, the dose of $5 \mathrm{mg}$ once a day was used, aspirin was stopped. Since there was no clinical sign of venous/arterial thrombosis, the patient was discharged from the Hospital on D23. A close follow-up by our outpatient clinic was performed. During fondaparinux treatment, platelet count slightly decreased: being $46 \times 10^{3} / \mu \mathrm{L}$ on D24 and D25, and $43 \times 10^{3} / \mu \mathrm{L}$ on D27. On D27, he complained of calf pain and swelling, a whole leg ultrasound revealed a thrombosis confined to the internal gastrocnemius vein and to the soleal vein in the symptomatic leg, that is, isolated distal DVT. Fondaparinux was stopped and rivaroxaban $15 \mathrm{mg}$ twice a day was started. As shown in -Fig. 1, the platelet count returned to baseline by 10 days following fondaparinux withdrawal. After 20 days since fondaparinux withdrawal, the patient was asymptomatic

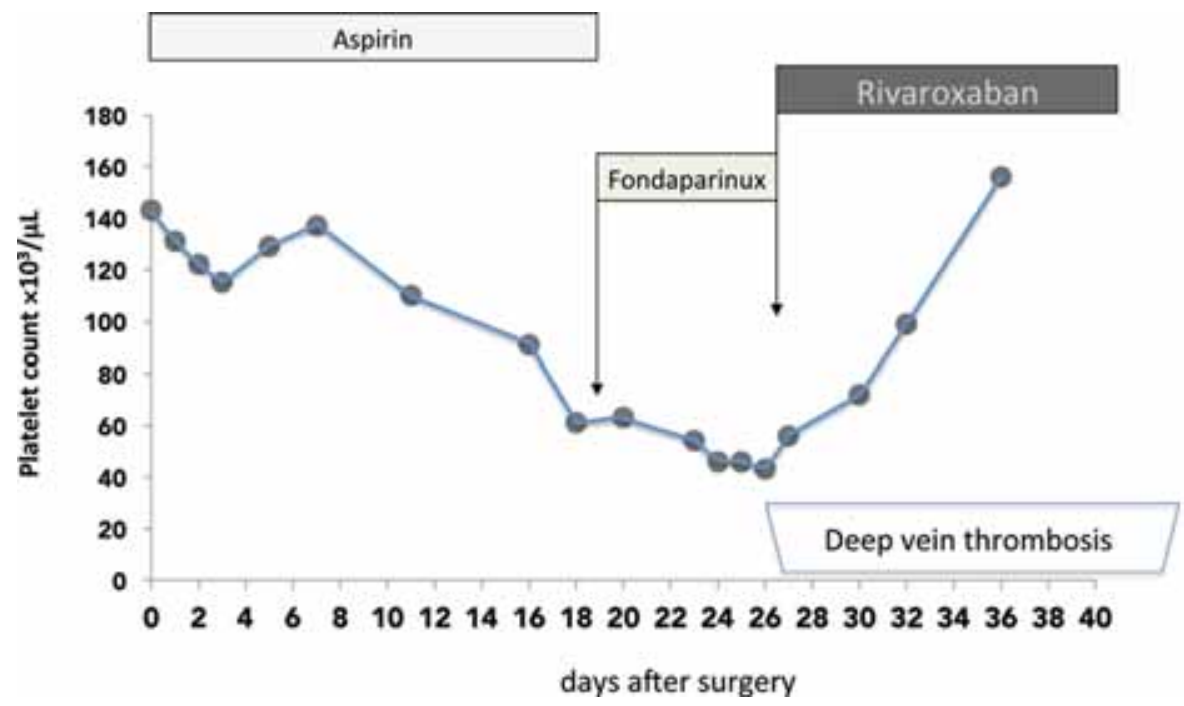

Fig. 1 Trend in platelet counts after carotid endarterectomy (D0: day 0). On D0 a bolus of heparin dose of 5,000 U was used, 27 days afterwards (D27) a thrombosis confined to the internal gastrocnemius vein and to the soleal vein occurred (deep vein thrombosis). Fondaparinux: fondaparinux $5 \mathrm{mg}$ once a day, Rivaroxaban: rivaroxaban $15 \mathrm{mg}$ twice a day. 
and the whole leg ultrasound showed recanalization of the calf DVT, rivaroxaban dosage was reduced to $20 \mathrm{mg}$ once a day, the gel immunoassay was still positive for PF4-heparin complexes. After 3 months, rivaroxaban was stopped, wholeleg ultrasound did not show any distal DVT or any proximal DVT and the gel immunoassay gave a negative result. In summary, the patient completed a total of 3 months of rivaroxaban therapy with no recurrent thrombotic or bleeding complications. During follow-up (1 year), no arterial, neither venous thrombosis occurred.

\section{Discussion}

Our experience suggests that fondaparinux should be used with caution in autoimmune HIT and, consistent with previous reports, rivaroxaban may be safely used to prevent new thrombosis in this hypercoagulable condition.

A recent German multicentre observational study showed no thrombotic complication in patient treated with fondaparinux ${ }^{5}$ supporting the use of fondaparinux in the everyday clinical practice for the treatment of suspected and confirmed HIT. ${ }^{12}$ Several limitation of the aforementioned study should be acknowledged: the study was retrospective, HIT was not confirmed in several patients, and the choice of the anticoagulant agent was left to the decision of the physician in charge. On the contrary, a retrospective study in English patients ${ }^{13}$ found a $16 \%$ thromboembolic rate in patients treated with fondaparinux for HIT. Here, we report a case of autoimmune HIT refractory to fondaparinux. Patients with autoimmune HIT seem to be at higher thrombotic risk than patients with HIT and often require a higher and stable therapeutic levels of anticoagulation. Since the presence of chronic kidney disease and since no thrombosis was found, fondaparinux at a dose of $5 \mathrm{mg}$ was used. It should be noted that the dose suggested for acute thromboembolism therapy is higher than the dose we used ( 5 vs. $7.5 \mathrm{mg}$ ), and this may explain the failure of fondaparinux in the present case. Moreover, it has been shown that fondaparinux may, although infrequently, cross-react with antiheparin PF4 antibodies, and at least theoretically provoke or exacerbate HIT. We did not measure fondaparinux cross reactivity with HIT antibodies, thus we can only speculate the cause of fondaparinux failure in the present case. It has to be noticed that, despite platelet started to decrease 11 days after surgery, HIT was suspected only after 16 days and anticoagulant treatment was started after 19 days. This delay may have lead to higher thrombotic burden and favored the occurrence of a new thrombosis.

The efficacy of rivaroxaban, an oral, direct factor Xa inhibitor, in the treatment of venous thromboembolism has been clearly established. ${ }^{14}$ Rivaroxaban does not show any interaction with PF4 or anti-PF4/heparin, ${ }^{15}$ and there is growing evidence that rivaroxaban can be an alternative anticoagulant agent in HIT, so far more than 100 patients with HIT have been treated with such agent with low thromboembolic and bleeding complications. ${ }^{9}$ During the past 4 years, we have already used rivaroxaban in other two cases of HIT, one has already been described, ${ }^{16}$ another was an HIT associated with DVT after orthopaedic surgery. We used rivaroxaban for HIT because it has been shown to be efficacious as single agent in the acute phase of DVT without a short initial parenteral anticoagulant treatment and it allowed us to treat the patient at home. We did not observed thromboembolic complications during rivaroxaban treatment in these three patients. It has to be noted that all the three patients whom we treated with rivaroxaban had HIT associated with thrombosis limited to the vein of lower leg. In our opinion, these patients may be a lower risk than patients with HIT associated with pulmonary embolism or arterial thrombosis.

Autoimmune HIT is caused by platelet-activating anti-PF4/ polyanion antibodies that are able to activate platelets in the absence of heparin. ${ }^{3}$ Autoimmune HIT encompasses several clinical conditions, including delayed-onset HIT, spontaneous HIT syndrome (HIT without proximate heparin exposure), flush heparin HIT (HIT induced by exposure to heparin flushes), fondaparinux-associated HIT (HIT triggered by exposure to fondaparinux), and is associated with persistent thrombocytopenia and often with disseminated intravascular coagulation. ${ }^{3}$ This condition requires treated with consistently therapeutic levels of anticoagulation. Our patient clearly had a diagnosis of autoimmune HIT based on the clinical picture of delayed onset and persisting thrombocytopenia following a single-intraoperative UFH exposure, as well as the laboratory demonstration of patient's serum-induced platelet aggregation that occurred even in the absence of heparin but which was inhibited by high concentrations of heparin.

Rivaroxaban is one of the treatment options recommended for treating the hypercoagulability state associated with autoimmune HIT. $^{3}$ Indeed, at least seven cases of

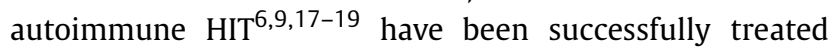
with rivaroxaban. In contrast, several reports ${ }^{6,7,17}$ have highlighted unsuccessful outcomes when fondaparinux was used as the first therapeutic agent for autoimmune HIT. There are at least two explanations why rivaroxaban, given $15 \mathrm{mg}$ twice a day, may be superior to fondaparinux in treating autoimmune HIT. First, twice-daily dosing of rivaroxaban versus once-daily fondaparinux dosing may better achieve persistent levels of therapeutic anticoagulation, and control of severe HIT-associated hypercoagulability. Second, autoimmune HIT may be a high-risk situation for fondaparinux cross-reactivity, a problem not seen with rivaroxaban.

\section{Limitations and Conclusion}

Some limitations of our report should be acknowledged. First, we were not able to determine whether fondaparinux cross-reactivity was a potential explanation for treatment failure in our patient with autoimmune HIT. Second, we cannot exclude the possibility that the explanation for fondaparinux failure was the dose administered (5 mg), which was lower than the usual dose $(7.5 \mathrm{mg}$ ) recommended for treatment of acute HIT-associated thromboembolism. However, it should be noted that the Food and Drug Administration (FDA) recommends that fondaparinux be used with caution in patients with creatinine clearance of 30 to $50 \mathrm{~mL} / \mathrm{min}$, and our patient had additional considerations 
(advanced age and concomitant antiplatelet medications). ${ }^{20}$ Thus, the $5 \mathrm{mg}$ dose we gave seemed appropriate for initial treatment in our 86-year-old male patient with chronic renal disease. Despite the additional limitation of a single case report, our observations are consistent with emerging clinical experience and supports the intriguing hypothesis that rivaroxaban may be a superior option over fondaparinux for treating autoimmune HIT.

\section{Note}

Informed consent was obtained from the patient for the purpose of publication.

\section{Funding}

None.

\section{Conflict of Interest \\ None declared.}

\section{References}

1 Cosmi B. Current management of heparin-induced thrombocytopenia. Expert Rev Hematol 2015;8(06):837-849

2 Greinacher A. Heparin-induced thrombocytopenia. J Thromb Haemost 2009;7(Suppl 1):9-12

3 Greinacher A, Selleng K, Warkentin TE. Autoimmune heparin-induced thrombocytopenia. J Thromb Haemost 2017;15(11):2099-2114

4 Cuker A, Arepally GM, Chong BH, et al. American Society of Hematology 2018 guidelines for management of venous thromboembolism: heparin-induced thrombocytopenia. Blood Adv 2018;2(22):3360-3392

5 Schindewolf M, Steindl J, Beyer-Westendorf J, et al. Use of fondaparinux off- label or approved anticoagulants for management of heparin- induced thrombocytopenia. J Am Coll Cardiol 2017;70 (21):2636-2648

6 Poudel DR, Ghimire S, Dhital R, Forman DA, Warkentin TE. Spontaneous HIT syndrome post-knee replacement surgery with delayed recovery of thrombocytopenia: a case report and literature review. Platelets 2017;28(06):614-620

7 Tvito A, Bakchoul T, Rowe JM, Greinacher A, Ganzel C. Severe and persistent heparin-induced thrombocytopenia despite fondaparinux treatment. Am J Hematol 2015;90(07):675-678
8 Linkins LA, Warkentin TE, Pai M, et al. Rivaroxaban for treatment of suspected or confirmed heparin-induced thrombocytopenia study. J Thromb Haemost 2016;14(06):1206-1210

9 Warkentin TE, Pai M, Linkins LA. Direct oral anticoagulants for treatment of HIT: update of Hamilton experience and literature review. Blood 2017;130(09):1104-1113

10 Lo GK, Juhl D, Warkentin TE, Sigouin CS, Eichler P, Greinacher A. Evaluation of pretest clinical score (4 T's) for the diagnosis of heparininduced thrombocytopenia in two clinical settings. J Thromb Haemost 2006;4(04):759-765

11 Legnani C, Cini M, Pili C, Boggian O, Frascaro M, Palareti G. Evaluation of a new automated panel of assays for the detection of anti-PF4/ heparin antibodies in patients suspected of having heparin-induced thrombocytopenia. Thromb Haemost 2010;104(02):402-409

12 Schindewolf M, Steindl J, Beyer-Westendorf J, et al. Frequent offlabel use of fondaparinux in patients with suspected acute heparin-induced thrombocytopenia (HIT)-findings from the GerHIT multi-centre registry study. Thromb Res 2014;134(01):29-35

13 Kang M, Alahmadi M, Sawh S, Kovacs MJ, Lazo-Langner A. Fondaparinux for the treatment of suspected heparin-induced thrombocytopenia: a propensity score-matched study. Blood 2015;125(06):924-929

14 Cohen AT, Bauersachs R. Rivaroxaban and the EINSTEIN clinical trial programme. Blood Coagul Fibrinolysis 2019;30(03):85-95

15 Krauel K, Hackbarth C, Fürll B, Greinacher A. Heparin-induced thrombocytopenia: in vitro studies on the interaction of dabigatran, rivaroxaban, and low-sulfated heparin, with platelet factor 4 and anti-PF4/heparin antibodies. Blood 2012;119(05): 1248-1255

16 Sartori M, Favaretto E, Cini M, Legnani C, Cosmi B. Rivaroxaban in the treatment of heparin-induced thrombocytopenia. J Thromb Thrombolysis 2015;40(03):392-394

17 Manji F, Warkentin TE, Sheppard JI, Lee A. Fondaparinux crossreactivity in heparin-induced thrombocytopenia successfully treated with high-dose intravenous immunoglobulin and rivaroxaban. Platelets 2020;31(01):124-127

18 Bakchoul T, Borst O, Riessen R, et al. Autoimmune heparininduced thrombocytopenia after transcatheter aortic valve implantation: successful treatment with adjunct high-dose intravenous immunoglobulin. TH Open 2019;3(02):e200-e202

19 Mian H, Warkentin TE, Sheppard JI, et al. Autoimmune HIT due to apheresis catheter heparin flushes for stem cell harvesting before autotransplantation for myeloma. Blood 2017;130(14):1679-1682

20 ARIXTRA. Available at: https://www.accessdata.fda.gov/drugsatfda_docs/label/2005/021345s010lbl.pdf. Accessed 28 April 2020 